\title{
Superficial Siderosis of the Central Nervous System due to Spinal Ependymoma
}

\author{
Ko-Eun Choi ${ }^{1}$, Seung-Hee $\mathrm{Na}^{1}$, Hyeonseok S. Jeong ${ }^{2}$, Jooyeon J. $\mathrm{Im}^{2}$, Young-Do Kim ${ }^{1}$ \\ Departments of ${ }^{1}$ Neurology and ${ }^{2}$ Radiology, Incheon St. Mary's Hospital, The Catholic University of Korea, Incheon, Korea
}

Corresponding Author: Young-Do Kim, MD, PhD

Department of Neurology, Incheon St. Mary's Hospital, The

Catholic University of Korea, 56 Dongsu-ro, Bupyeong-gu, Incheon 21431, Korea

Tel: $+82-32-280-5010$

Fax: +82-32-280-6390

E-mail: limbic@catholic.ac.kr

Received: January 11, 2018

Revised: March 6, 2018

Accepted: March 13, 2018
A 75-year-old woman presented with a 3-year history of progressive hearing loss, gait ataxia, and cognitive impairment. Brain magnetic resonance imaging (MRI) with a time gradient echo sequence showed deposition of hemosiderin along the surface of the cerebral cortex, brainstem, and cerebellum, as well as severe atrophy in the diffuse cerebral cortex and cerebellum. We established the diagnosis of superficial siderosis of the central nervous system on the grounds of former pathognomonic MRI findings. The thoraco-lumbar spine MRI demonstrated a myxopapillary ependymoma in the T11-L2 spinal canal that was considered to be the cause of a chronic subarachnoid hemorrhage, affecting the leptomeninges and subpial layers of the central nervous system.

Key Words: Siderosis, Ependymoma, Spinocerebellar ataxia

\section{INTRODUCTION}

Superficial siderosis of the central nervous system (SSCNS) is a rare disease resulting from the accumulation of hemosiderin in the meninges, brain surface, spinal cord, and cranial nerves. SSCNS presents as slowly progressive sensorineural hearing loss due to involvement of the 8th cranial nerve, gait ataxia, pyramidal signs, and cerebellar dysfunction. ${ }^{1)}$ Despite extensive laboratory, imaging, and clinical evaluations, the bleeding site remains unidentified in approximately half of the cases. ${ }^{1,2)}$ In this report, we describe a patient with hearing impairment, ataxia, and cognitive decline owing to SSCNS caused by spinal ependymoma-induced subarachnoid bleeding.

\section{CASE REPORT}

A 75-year-old woman was admitted due to the presence of progressive hearing loss, gait ataxia, and cognitive impairment over a span of 3 years. She also complained of severe back pain and difficulty in urinating and defecating. We confirmed her bilateral sensorineural hearing loss using pure tone audiometry. Moreover, a neurological examination revealed dysarthria, dysphagia, bilateral lower extremity weakness, severe gait disturbance, dysmetria, dysdiadochokinesia, and intention tremor, suggesting cerebellar involvement. Further examination revealed hyperreflexia in 4 limbs and ankle clonus, which were indicative of bilateral pyramidal tract lesion.

Owing to the patient's bilateral deafness, it was difficult to conduct a detailed cognitive assessment. Nevertheless, we observed severe language dysfunction, memory impairment, bizarre behavior, and depressive mood. Her past medical and surgical history was unremarkable except for long-standing back pain and difficulty in urinating. Brain magnetic resonance imaging (MRI) with a time gradient echo (GRE) sequence showed diffuse linear hypointensity (typical black rim) along the surface of the cerebral cortex, Sylvian fissure, brainstem, and cerebellum compatible with extensive deposition of hemosiderin, as well as severe atrophy in the diffuse cerebral cortex and cerebellum (Fig. 1A-D). These imaging findings strongly suggested that there was a chronic subarachnoid hemorrhage affecting the leptomeninges and subpial layers of the central nervous system. ${ }^{3)}$ We established the diagnosis of SSCNS based on previously reported MRI findings. ${ }^{2,45}$ We excluded various coagulation disorders as a cause of the chronic subarachnoid hemorrhage through further laboratory evaluation. Brain MR angiography was performed to determine the cause of the subarachnoid hemorrhage. It exhibited normal findings. The thoraco-lumbar spine MRI 

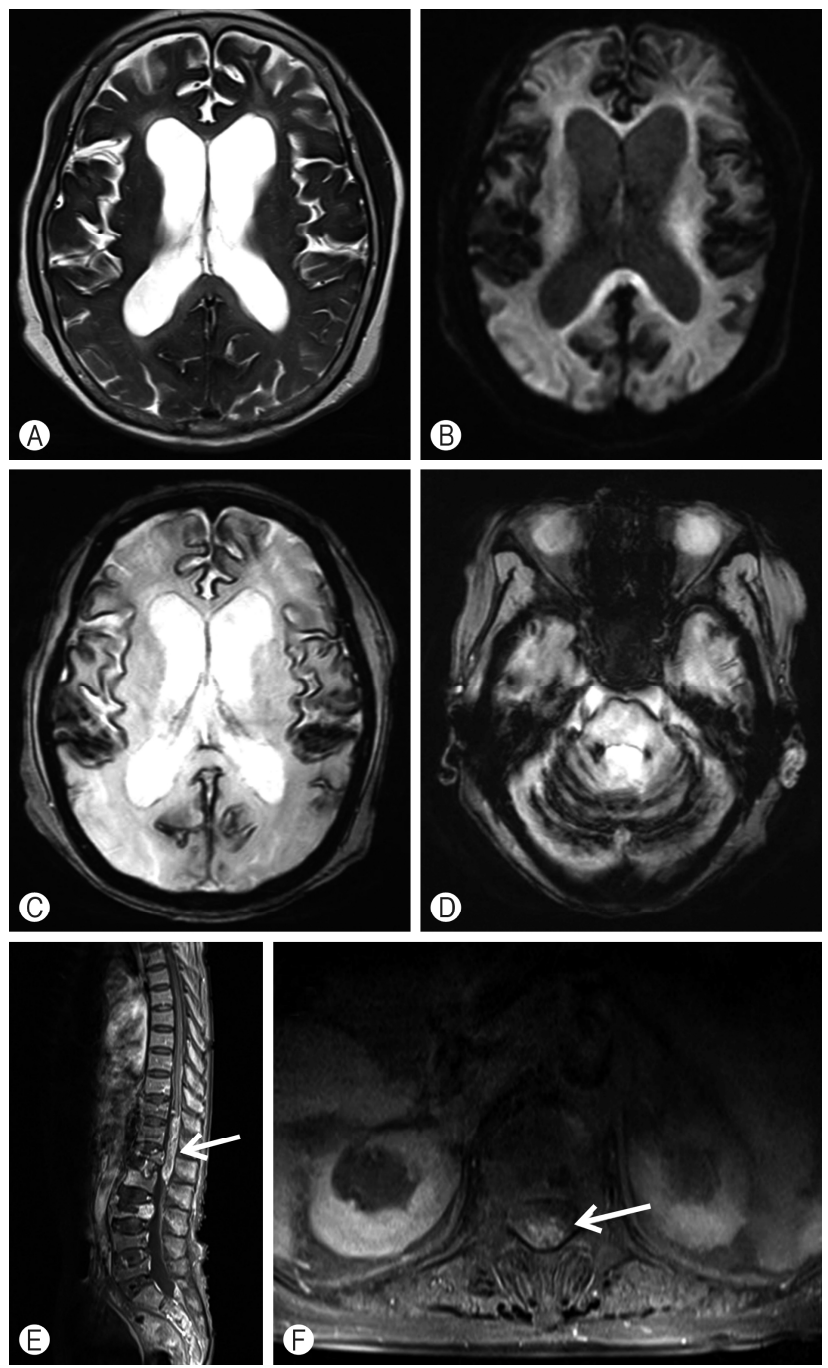

Fig. 1. Axial T2-weighted (A) and diffusion-weighted (B) images showing a low signal rim of hemosiderin surrounding the cerebral sulci. Definite linear hypointensity is seen along the Sylvian fissure, cerebral sulci, cerebellar folia, and superior vermis on the axial gradient echo images, compatible with extensive hemosiderin deposition. (C, D) The radiological findings are pathognomonic of superficial siderosis. Postgadolinium T1-weighted magnetic resonance imaging of the thoraco-lumbar spine shows an ovalshaped heterogeneous and highly enhanced $1.7 \times 1.2 \times 7-\mathrm{cm}$ mass (thick white arrows) in the T11-L2 spinal canal. (E, F) The radiological findings are compatible with myxopapillary ependymoma.

taken because of the patient's severe back pain demonstrated a myxopapillary ependymoma in the T11-L2 spinal canal (Fig. $1 \mathrm{E}, \mathrm{F})$ that was considered to be the cause of a chronic subarachnoid hemorrhage affecting extensive regions of the central nervous system. However, we were unable to perform a tumor removal operation owing to the refusal of a caretaker and the high-risk nature of the operation. The patient's symptoms became more aggravated and she became bedridden. Eventually, she was transferred to a nursing home.

\section{DISCUSSION}

The most common causes of SSCNS include ruptured aneurysms, arteriovenous malformation, intradural cranial or spinal surgery, traumatic injury, and bleeding of unknown etiology." However, more unusual causes of chronic bleeding include dural pathologies and tumors. ${ }^{1,3,6)}$ Tumors, including ependymomas, meningiomas, oligodendrogliomas, and pineocytomas, are found in 35\% of cases. ${ }^{1)}$ In our patient, the cause of SSCNS was ependymoma of the spinal cord identified as the bleeding source. If our patient had not complained of severe back pain, the cause of her SSCNS might not have been found. Therefore, to find the cause of SSCNS, careful history taking and neurological examination are essential. Hearing loss, cerebellar ataxia, and pyramidal signs are the most frequent clinical findings, occurring in $95 \%$, $88 \%$, and $76 \%$ of cases, respectively. ${ }^{3)}$ The long glial segment of the vestibulocochlear nerve makes it vulnerable to axonal damage by iron deposition. ${ }^{2)}$

The constellation of clinical features and the various degrees of symptom severity can make the diagnosis of SSCNS rather difficult. As in the present case, late-onset spinocerebellar ataxia might be considered in the differential diagnosis of adults with insidiously progressing gait disturbance, cognitive decline, and pyramidal and cerebellar signs. ${ }^{7)}$ There is a high risk of various wrong diagnoses, particularly in older patients, because of the slowly progressing and relatively common symptoms associated with SSCNS. Although brain MRI is the most important investigation method, the hypointensity in brain MRI can easily be missed in the early stages of SSCNS. GRE T2-weighted images have a higher sensitivity for hemosiderin deposition. 1) Therefore, GRE-MRI should be included in the standard imaging protocol when evaluating patients with a combination of hearing impairment, cognitive decline, and pyramidal and cerebellar signs, as was found in our patient. ${ }^{5)}$ Furthermore, because treatment may be possible, an aggressive effort is needed to locate the cause of SSCNS.

The limitation of our case is that the myxopapillary ependymoma was diagnosed by spinal MRI and not by histopathologic examination. Myxopapillary ependymoma is a rare, World Health Organization grade I ependymoma occurring predominantly in the lumbosacral region, particularly in the filum terminale. ${ }^{6}$ SSCNS caused by spinal ependymoma has only rarely been reported in the literature. ${ }^{5,89}$ Ependymoma presents as soft, encapsulated masses with a propensity to hemorrhage. ${ }^{8)}$ Therefore, ependymoma is a frequent cause of chronic subarachnoid bleeding. The surgical treatment of SSCNS depends on the early identification of the bleeding source, such as the offending neoplasm, vascular malformation, or pseudomeningocele. ${ }^{1)}$ Therefore, in our case, neurosurgical intervention of the spinal ependymoma could have 
been an effective therapy for SSCNS. 5,6,10)

In conclusion, we reported a case of SSCNS caused by a spinal ependymoma. SSCNS must be included in the differential diagnosis for combined progressive hearing impairment, cognitive impairment, ataxia, and pyramidal and extrapyramidal signs. Early diagnosis using GRE-MRI is important, and an effort should be made to find the bleeding source because treatment may be possible.

Conflicts of Interest Disclosures: The researchers claim no conflicts of interest.

\section{REFERENCES}

1. Kumar N. Superficial siderosis: associations and therapeutic implications. Arch Neurol 2007;64:491-6.

2. Kumar N, Cohen-Gadol AA, Wright RA, Miller GM, Piepgras DG, Ahlskog JE. Superficial siderosis. Neurology 2006;66:114452.

3. Fearnley JM, Stevens JM, Rudge P. Superficial siderosis of the central nervous system. Brain 1995;118(Pt 4):1051-66.
4. Kumar N. Neuroimaging in superficial siderosis: an in-depth look. AJNR Am J Neuroradiol 2010;31:5-14.

5. Spengos K, Vassilopoulou S, Tsivgoulis G, Karachalios G, Vassilopoulos D. Superficial siderosis due to a lumbar ependymoma mimicking adult-onset spinocerebellar ataxia. Clin Neurol Neurosurg 2007;109:705-7.

6. Pikis S, Cohen JE, Vargas AA, Gomori JM, Harnof S, Itshayek E. Superficial siderosis of the central nervous system secondary to spinal ependymoma. J Clin Neurosci 2014;21:2017-9.

7. Margolis RL. The spinocerebellar ataxias: order emerges from chaos. Curr Neurol Neurosci Rep 2002;2:447-56.

8. Grech R, Galvin L, Looby S, Thornton J. Spinal ependymoma complicated by superficial siderosis. BMJ Case Rep 2013 Sep 24;2013. pii: bcr2013201036. https://doi.org/10.1136/bcr-2013201036.

9. Salem A, Krainik A, Helias A, Bouccara D, Gaillard S, Feydy A, et al. MRI findings in a case of a superficial siderosis associated with an ependymoma. J Neuroradiol 2002;29:136-8.

10. Nakamura M, Ishii K, Watanabe K, Tsuji T, Matsumoto M, Toyama Y, et al. Long-term surgical outcomes for myxopapillary ependymomas of the cauda equina. Spine (Phila Pa 1976) 2009; 34:E756-60. 